\title{
On the efficiency of rocket-borne particle detection in the mesosphere
}

\author{
J. Hedin ${ }^{1}$, J. Gumbel ${ }^{1}$, and M. Rapp ${ }^{2}$ \\ ${ }^{1}$ Department of Meteorology, Stockholm University, 10691 Stockholm, Sweden \\ ${ }^{2}$ Leibniz Institute of Atmospheric Physics, Schloss-Str. 6, 18225 Kühlungsborn, Germany
}

Received: 21 November 2006 - Published in Atmos. Chem. Phys. Discuss.: 25 January 2007

Revised: 21 June 2007 - Accepted: 29 June 2007 - Published: 16 July 2007

\begin{abstract}
Meteoric smoke particles have been proposed as a key player in the formation and evolution of mesospheric phenomena. Despite their apparent importance still very little is known about these particles. Important questions concern the smoke number density and size distribution as a function of altitude as well as the fraction of charged particles. Sounding rockets are used to measure smoke in situ, but aerodynamics has remained a major challenge. Basically, the small smoke particles tend to follow the gas flow around the payload rather than reaching the detector if aerodynamics is not considered carefully in the detector design. So far only indirect evidence for the existence of meteoric smoke has been available from measurements of heavy charge carriers. Quantitative ways are needed that relate these measured particle population to the atmospheric particle population. This requires in particular knowledge about the sizedependent, altitude-dependent and charge-dependent detection efficiency for a given instrument. In this paper, we investigate the aerodynamics for a typical electrostatic detector design. We first quantify the flow field of the background gas, then introduce particles in the flow field and determine their trajectories around the payload structure. We use two different models to trace particles in the flow field, a Continuous motion model and a Brownian motion model. Brownian motion is shown to be of basic importance for the smallest particles. Detection efficiencies are determined for three detector designs, including two with ventilation holes to allow airflow through the detector. Results from this investigation show that rocket-borne smoke detection with conventional detectors is largely limited to altitudes above $75 \mathrm{~km}$. The flow through a ventilated detector has to be relatively large in order to significantly improve the detection efficiency.
\end{abstract}

\footnotetext{
Correspondence to: J. Hedin

(jonash@misu.su.se)
}

\section{Introduction}

There is an increasing interest in meteoric material in the mesosphere. Most meteoroid mass entering the Earth's atmosphere vaporizes during atmospheric entry (Ceplecha et al., 1998). The total amount of incoming material is still controversial with estimates varying typically between 10 and 100 tons per day (Love and Brownlee, 1993; Mathews et al., 2001; von Zahn, 2005). It is well recognized that meteoroid ablation is the source of the metal atom layers that are observed by lidars and satellites, and much progress has been made in understanding the chemistry of these metals (Plane, 2003). More conjecture is the subsequent fate of the material. Chemical conversion, re-condensation and coagulation of the evaporated species is thought to generate meteoric smoke particles in the nanometre size range (Rosinski and Snow, 1961; Hunten et al., 1980; Megner et al., 2006). Although there is today growing experimental evidence for the existence of such particles, little is known about their actual properties and atmospheric distribution.

Despite of these uncertainties, meteoric smoke has been proposed as a key player in the generation and evolution of mesospheric phenomena. Smoke particles can provide condensation nuclei for ice particles involved in noctilucent clouds (NLC) and polar mesosphere summer echoes (PMSE) (Rapp and Thomas, 2006). Smoke particles have been suggested as a surface for heterogeneous chemistry in the mesosphere, influencing e.g. the water vapour budget (Summers and Siskind, 1999). Smoke particles are thought to serve as ultimate sink for mesospheric metal chemistry (Plane, 2004). Being part of the ionosphere, smoke particles also participate in the charge balance by giving rise to a "dusty plasma" in the D-region (Rapp and Lübken, 2001). In addition to these interactions in the mesosphere, smoke particles could play important roles in the formation of polar stratospheric clouds (Voigt et al., 2005) and as a tracer of atmospheric circulation in ice cores (Gabrielli et al., 2004; Lanci and Kent, 2006).

Published by Copernicus Publications on behalf of the European Geosciences Union. 
Given all these potential relationships, it is obvious that there is a large scientific interest in the properties and global distribution of meteoric smoke. However, the observational data base is sparse, a fact that is related to the experimental difficulties in detecting smoke. Based on our current knowledge, smoke particles are too small for optical detection and their momentum is not sufficient to generate detectable acoustical or electrical pulses upon impact. For this reason, the experimental study of mesospheric smoke has largely been limited to in situ charge-sensitive measurements of the charged fraction of these particles.

The major class of detectors aimed at the detection of charged meteoric smoke is based on a detector design originally developed by Havnes et al. (1996) for the study of ice particles in the polar summer mesosphere. The detector concept uses a Faraday cup for the detection of incoming heavy charge carriers in combination with biased grids that shield against contamination by electrons and light ions from the ambient D-region. The first application of such a detector to the rocket-borne study of meteoric smoke was by Gelinas et al. (1998). Lynch et al. (2005) further developed this detector design to also allow a discrimination between positive and negative particles. Rapp et al. (2005) combined the classical cup design of Havnes et al. (1996) for the detection of charged particles with a xenon flash lamp for the detection of neutral atmospheric particles by photoionization. Altogether, smoke data from Faraday-type detectors is today available from seven rocket flights. The current paper focuses on a closer investigation of this detector type.

In addition to the Faraday detectors considered here, other techniques have been applied to study meteoric smoke. Heavy charged constituents have been measured from sounding rockets by Schulte and Arnold (1992) using a mass spectrometer, by Croskey et al. (2001) using a Gerdien condenser, and by Robertson et al. (2004) and Smiley et al. (2006) using magnetically shielded probes. Signatures of charged particles have recently also been reported from incoherent scatter radar data (Rapp et al., 2007). It is important to note that none of the above measurements of heavy charge carriers provides a definite proof that the detected species really are smoke particles of meteoric origin. In order to provide such a proof and more detailed studies of the particles, instruments have been flown to directly sample mesospheric smoke (Gumbel et al., 2005). Results from these investigations have not been published yet.

Important scientific questions concern the number density and size distribution of smoke particles as a function of altitude, but also their composition, charge state and interaction with the neutral atmosphere and ionosphere. Sounding rockets are the only means of studying smoke particles in situ in the mesosphere. But these measurements are difficult and a number of challenges inherent to sounding rocket experiments need to be considered, such as aerodynamics and charging processes. The interpretation of mesospheric particle measurements requires a detailed understanding of the detector response, which is far from trivial. Basic instrumental questions are:

- How are measured particle concentrations related to the undisturbed particle concentrations in the atmosphere?

- How are properties of detected particles related to the particle properties in the atmosphere?

- How is the charge measured by particle detectors related to the charge of particles in the atmosphere?

In the current paper, we focus on aerodynamic effects that have a potential influence on all of these questions. For a given instrument design, our aim is to provide a response function, specifying the fraction of atmospheric particles that is actually detected as a function of particle size and charge, altitude, and flow conditions.

The basic aerodynamic challenge lies in the size of the meteoric smoke particles. The particles are so small that they tend to follow the gas flow around the payload rather than reaching the detector. Since we want the particles to hit the detector surface, careful aerodynamic design is thus of critical importance for smoke experiments. Numerical simulations of particle impact are conveniently modelled in two steps. First, the flow field of the background gas needs to be quantified (Gumbel, 2001a); second, particles are introduced in the flow field and their trajectories around the payload structure are determined. Simulations of rocketborne measurements of smoke particles and ice condensates in the mesosphere have first been considered by Horányi et al. (1999). We present results based on their model ideas, describing the interaction between gas and particles by a continuous drag force. We then introduce a model that takes into account the Brownian motion of particles in the gas. The resulting flow patterns are closer to the real motion of smoke particles, which is especially important for the smallest particles.

The model for the rarefied gas flow and the two models for the flow of smoke particles are described in Sect. 2. Based on a typical Faraday detector geometry, Sect. 3 then provides results on particle detection efficiencies and discusses the importance of the aerodynamic design. Section 4 summarizes with conclusions and an outlook. An appendix is added to explain the Brownian motion model in more detail.

\section{Model descriptions}

\subsection{Gas flow}

The aerodynamic analysis of mesospheric particle measurements is complicated by the fact that the rocket payload moves through different flow regimes. Between 50 and $130 \mathrm{~km}$, conditions change from continuum flow via the transition regime to free molecular flow. The rarefaction of the gas is conveniently described by the Knudsen number $K n$ 
which relates the atmospheric mean free path $\lambda$ to a characteristic dimension $L$ of the rocket payload or instrument:

$$
K n=\frac{\lambda}{L}
$$

The freestream mean free path is, assuming a hard-sphere description of molecular collisions in the gas, inversely proportional to the molecular number density $n$ (Bird, 1994):

$\lambda=\frac{1}{\sqrt{2} \sigma n}$,

where the collision cross section for air is $\sigma \approx 4.3 \times 10^{-19} \mathrm{~m}^{2}$. In the continuum flow regime, with $K n<0.1$, conventional tools of computational fluid dynamics are applicable. In the free molecular flow regime, $K n>>10$, an analysis is possible by assuming collisionless paths of individual molecules. However, in the transition regime in-between, molecular collisions are neither negligible nor frequent enough to regard the gas as a continuum. Direct Simulation Monte Carlo (DSMC) models have become a common tool for the study of rarefied gas dynamics (Bird, 1994). We use the DS2V model by Bird which simulates two-dimensional and axially symmetric problems from continuum flow conditions to free molecular flow. The DSMC model is a microscopic approach, it analyses the behaviour of individual gas molecules. Typically $\sim 10^{5}$ representative molecules are simultaneously traced through the volume of interest. Basic inputs are the properties of the undisturbed gas flow (e.g. the number density, temperature and mean flow velocity) and the relevant properties of the payload (e.g. the geometry, surface temperature and reflection properties). In the model, collisions with other molecules and with payload surfaces are performed in accordance with suitable parameterizations and optimized in terms of numerical efficiency and accuracy.

DSMC is a direct simulation of the microphysical processes in a gas flow as compared to conventional computational fluid dynamics where solutions to macroscopic equations are sought. Steady state conditions are approached for large times and macroscopic flow properties like density, temperature and velocity fields are obtained by appropriate averaging of the molecular behaviour. Examples of DSMC applications to the analysis of mesospheric sounding rocket experiments are Bird (1988), Gumbel (2001a, b), Croskey et al. (2001), Rapp et al. (2001, 2005) and Hedin et al. (2005). Figure 1 show the normalised number density field of the background gas around the three designs of the Faraday Cup used in this study. The three cup designs are identical except that one of these designs is closed and the other two are ventilated to improve the aerodynamic properties of the detector.

\subsection{Continuous motion model}

To simulate particle impacts on detector surfaces, two models have been developed to introduce meteoric smoke particles in
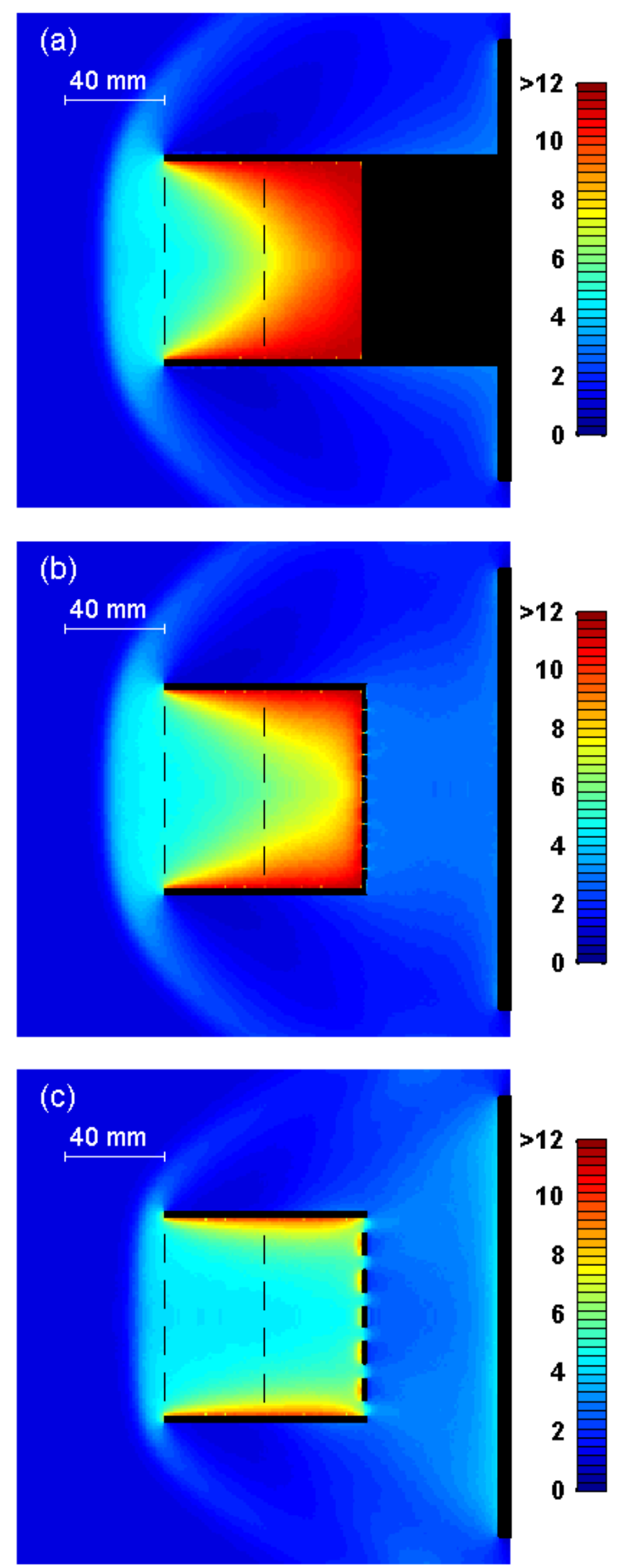

Fig. 1. Density field from the DSMC model of the background gas around three detector designs at $75 \mathrm{~km}$ altitude normalized to the undisturbed freestream density. The detector designs are (a) unventilated, (b) ventilated with $10 \%$ transmission, and (c) ventilated with $50 \%$ transmission. The flow is from the left with a speed of $1000 \mathrm{~m} / \mathrm{s}$. 

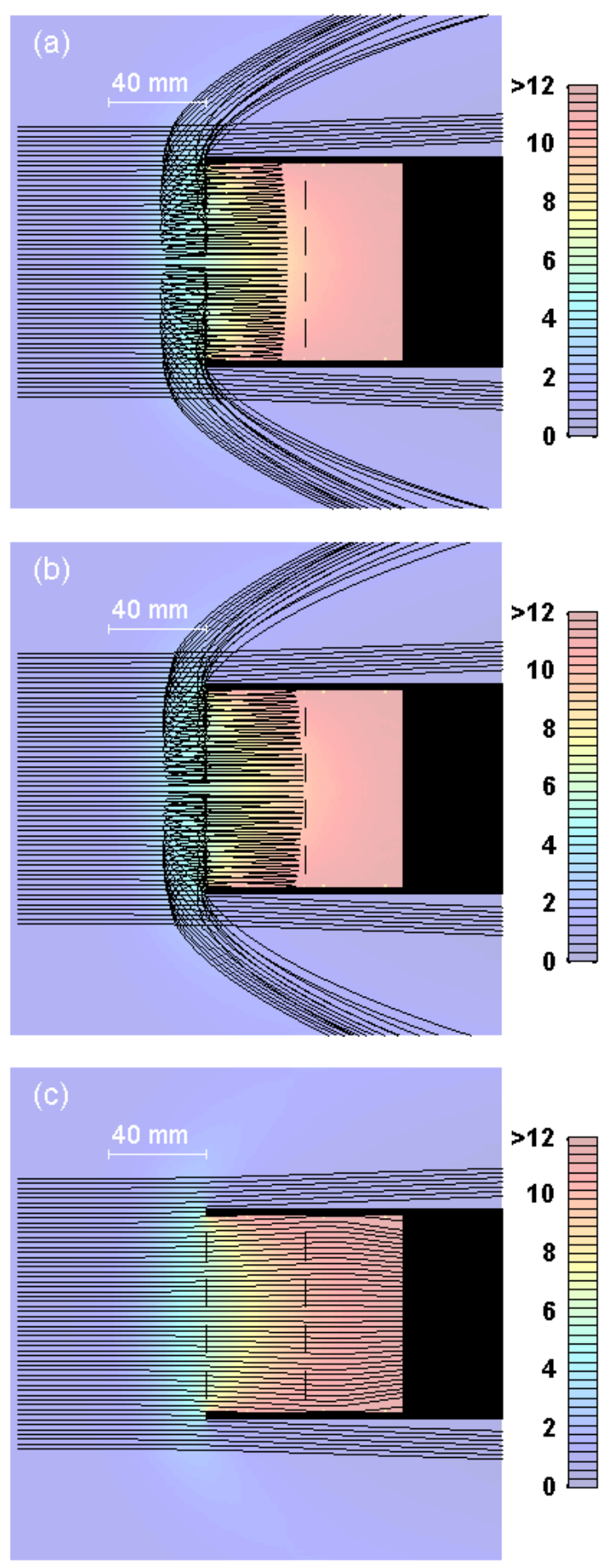

Fig. 2. Continuum motion model of (a) 1.0, (b) 1.1 , and (c) $1.2 \mathrm{~nm}$ radius positively charged particles approaching the unventilated detector at an altitude of $85 \mathrm{~km}$. The flow is from the left.

the gas flow and to determine their trajectories around payload structures: the Continuous motion model and the Brownian motion model.
Simulations of rocket-borne measurements of smoke particles and ice condensates in the mesosphere have first been considered by Horányi et al. (1999) and the Continuous motion model is based on their work. In the mesosphere and lower thermosphere region, particles of radii exceeding $\sim 10 \mathrm{~nm}$ experience sufficiently many collisions that the momentum transfer from the gas may be regarded as continuous. This makes it possible to write the equation of motion for particles as (Horányi et al, 1999; Probstein, 1968)

$$
\frac{4 \pi}{3} \rho_{p} r_{p}^{3} \frac{d \boldsymbol{v}_{p}}{d t}=r_{p}^{2} \pi \frac{C_{D}}{2} N_{g} m_{g}\left|\overline{\boldsymbol{V}}_{g}-\boldsymbol{v}_{p}\right|\left(\overline{\boldsymbol{V}}_{g}-\boldsymbol{v}_{p}\right)
$$

where $\rho_{p}, r_{p}$ and $\boldsymbol{v}_{p}$ are the density, radius and velocity of the smoke particle respectively. We assume a particle density of $3 \mathrm{~g} / \mathrm{cm}^{3}$ which is typical for chondritic material (Ceplecha et al., 1998). $C_{D}$ is the drag coefficient and can be calculated assuming that the incident gas molecules leave the surface of the particle diffusively with a Maxwellian velocity distribution set by the particles surface temperature (Probstein, 1968). $N_{g}$ and $\overline{\boldsymbol{V}}_{g}$ are the number density of the gas surrounding the particle and the mean flow velocity of the gas molecules, respectively, given by the DSMC model, and $m_{g}$ is the mean mass of an air molecule ( $29 \mathrm{amu})$. Mass loss due to heating and subsequent sublimation in the shocked gas flow is not considered. As opposed to ice particles, these effects are negligible for meteoric smoke (Horányi et al., 1999).

In the model, all particles start at an appropriate distance ahead of the detector or payload structure with a velocity relevant to the flight conditions of interest. The particles are then traced until they either hit the payload or leave the simulated area. Figure 2 shows results from the Continuous motion model of trajectories for positively charged particles of $1.0,1.1$, and $1.2 \mathrm{~nm}$ radius at an altitude of $85 \mathrm{~km}$ approaching the detector in Fig. 1a.

\subsection{Brownian motion model}

The Brownian motion model is a Monte Carlo model based on the statistical motion of the dust particles due to collisions with thermal air molecules in the air flow. In rarefied gas conditions these collisions can be regarded as binary just involving one particle and one molecule at a time. We treat the collision partners as hard spheres, i.e. there is no interchange of internal energy. Again, mass loss due to evaporation can be neglected for meteoric smoke. The basic model task is then to describe a particle's random path through the gas by performing representative collisions with the molecules. This involves several random steps: First a colliding molecule is chosen with a velocity $\boldsymbol{v}_{g}$ in accordance with the local flow conditions. Second, the collision is performed resulting in a new particle velocity $\boldsymbol{v}_{p}^{\prime}$. Third, a representative time period $\tau_{\text {coll }}$ is chosen until the next collision. These three steps are described in the following. 


\subsubsection{Choice of $\boldsymbol{v}_{g}$}

The velocity distribution of molecules colliding with a particle depends on the relative velocity $\overline{\boldsymbol{V}}_{\text {rel }}$ between particle and mean gas flow as well as the local temperature $T$. For an appropriate range of $\bar{V}_{\text {rel }}$ and $T$, velocity distributions are pre-calculated from simple gas kinetics and stored in lookup tables. These look-up tables are then used during the simulations to choose a colliding molecular velocity. First a collision angle $\theta$ with respect to the direction of $\overline{\boldsymbol{V}}_{\text {rel }}$ is randomly picked from a 2-dimensional look-up table in $\bar{V}_{\text {rel }}$ and $T$. Then a collision speed $v_{g}$ is randomly picked from a 3-dimensional look-up table in $\bar{V}_{\text {rel }}, T$ and $\theta$. The third component of $\boldsymbol{v}_{g}$, the azimuth angle around the direction of $\overline{\boldsymbol{V}}_{\text {rel }}$, is chosen isotropically between 0 and $2 \pi$. Finally, after having been determined with respect to the direction of $\overline{\boldsymbol{V}}_{\text {rel }}, \boldsymbol{v}_{g}$ is rotated into the payload coordinate system by an appropriate coordinate transformation.

\subsubsection{Choice of $\boldsymbol{v}_{p}^{\prime}$}

The velocity of the particle is then changed by the collision with this randomly chosen air molecule. Using the momentum and energy equations for the collision, the post-collision velocity of the particle can be derived (see Appendix A1) as

$\boldsymbol{v}_{p}^{\prime}=\boldsymbol{v}_{m}-\frac{m_{g}}{m_{g}+m_{p}} \boldsymbol{v}_{\text {rel }}^{\prime}$

where $\boldsymbol{v}_{m}$ is the velocity of the centre of mass of the two collision partners, $m_{g}$ and $m_{p}$ are the masses of the molecule and particle, respectively, and $\boldsymbol{v}_{\text {rel }}^{\prime}$ is the relative velocity between the molecule and particle after the collision. Both $\boldsymbol{v}_{m}$ and $\boldsymbol{v}_{\text {rel }}$ can be calculated from the pre-collision velocities $\boldsymbol{v}_{p}$ and $\boldsymbol{v}_{g}$. The magnitude of the relative velocity is unchanged by the collision, i.e. $v_{\text {rel }}^{\prime}=v_{\text {rel }}$. The direction of the relative velocity is distributed isotropically for spherical collision partners and, hence, the relative velocity after the collision is

$\boldsymbol{v}_{\mathrm{rel}}^{\prime}=v_{\mathrm{rel}}^{\prime} \hat{\boldsymbol{e}}=v_{\mathrm{rel}} \hat{\boldsymbol{e}}$

with an isotropically chosen direction $\hat{\boldsymbol{e}}$ (see Appendix A1).

\subsubsection{Choice of $\tau_{\text {coll }}$}

The mean time $\bar{\tau}_{\text {coll }}$ between two collisions is determined by the local air number density and mean collision speed $\bar{v}_{\text {coll }}$ (see Appendix A2). In accordance with this $\bar{\tau}_{\text {coll }}$, a random time period between two subsequent collisions can be chosen as

$\tau_{\text {coll }}=-\ln \left(r_{3}\right) \times \bar{\tau}_{\text {coll }}$

where $r_{3}$ is a random number, $0<r_{3} \leq 1$. The remaining $\tau_{\text {coll }}$ until the next collision is determined at each new particle position. The model time step $\Delta t$ is generally set to be shorter than the mean collision time in the unperturbed atmosphere. After a collision, or a series of collisions, has been performed
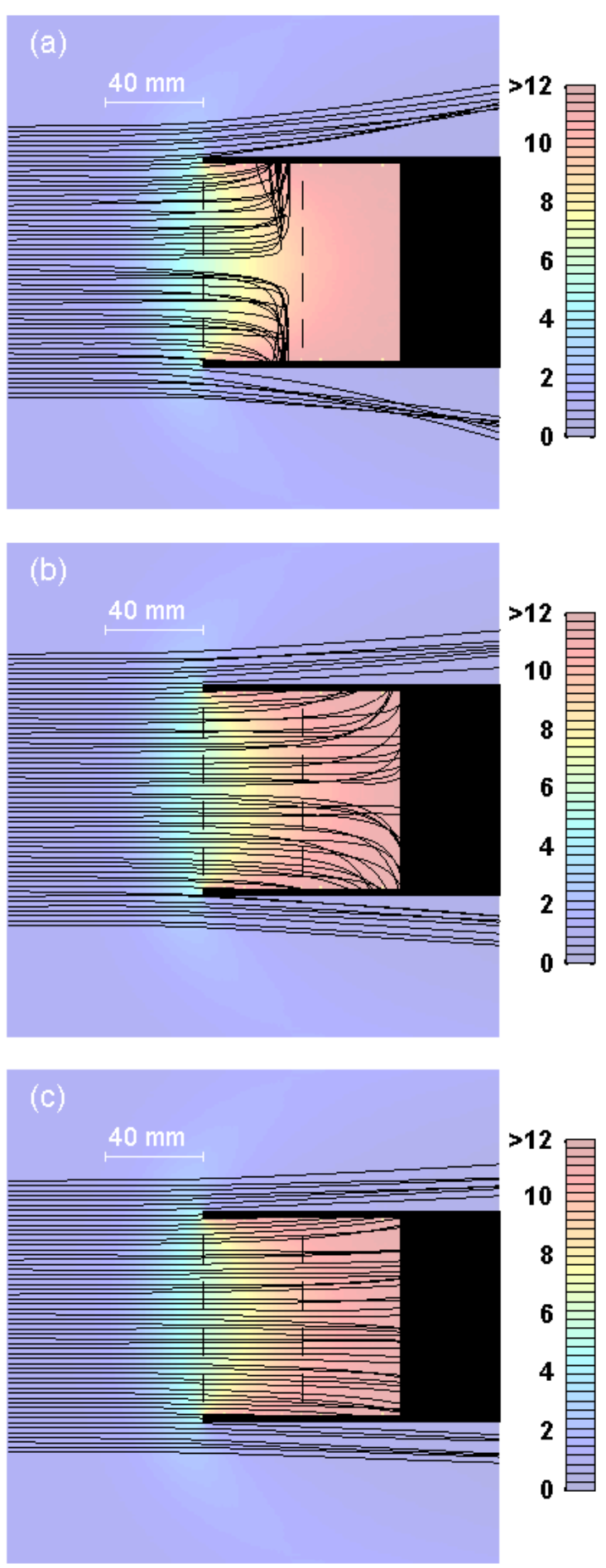

Fig. 3. Brownian motion model of (a) 1.5 , (b) 2.0 and (c) $2.5 \mathrm{~nm}$ radius positively charged particles approaching the unventilated detector at an altitude of $85 \mathrm{~km}$.

at a certain point the particle will move collisionless for one or several time steps $\Delta t$ until $\tau_{\text {coll }}$ is reached and the next collision is performed. Collisions are performed and the 
Table 1. Air number densities, temperatures and rocket velocity for the simulated altitudes.

\begin{tabular}{cccc}
\hline $\begin{array}{c}\text { Alt. } \\
(\mathrm{km})\end{array}$ & $\begin{array}{c}\text { Number density } \\
\left(\mathrm{m}^{-3}\right)\end{array}$ & $\begin{array}{c}\text { Temp. } \\
(\mathrm{K})\end{array}$ & $\begin{array}{c}\text { Velocity } \\
(\mathrm{m} / \mathrm{s})\end{array}$ \\
\hline 95 & $2.6 \times 10^{19}$ & 192 & 1000 \\
90 & $6.1 \times 10^{19}$ & 203 & 1000 \\
85 & $1.2 \times 10^{20}$ & 211 & 1000 \\
80 & $2.8 \times 10^{20}$ & 225 & 1000 \\
75 & $6.0 \times 10^{20}$ & 228 & 1000 \\
70 & $1.1 \times 10^{21}$ & 231 & 1000 \\
\hline
\end{tabular}

particles are traced until they either hit the payload structure or leave the simulated area. As an example, Fig. 3 show particle trajectories for $1.5,2.0$ and $2.5 \mathrm{~nm}$ radius particles modelled by the Brownian motion model around the unventilated detector at an altitude of $85 \mathrm{~km}$. Note that while all particle/molecule collisions are performed in three dimensions, the plots in Fig. 3 are two-dimensional with the particle trajectories projected back into the $\phi=0^{\circ}$ plane of the cylindrical coordinates.

\section{Results and discussion}

The modelling of the background gas flow around the detector was made for a winter atmosphere at $68^{\circ}$ north latitude with air number density and temperature taken from the MSIS-E-90 model (Hedin, 1991) at six different altitudes $70,75,80,85,90$ and $95 \mathrm{~km}$ (see Table 1). The velocity of the modelled rocket payload was set to $1000 \mathrm{~m} / \mathrm{s}$ over the entire altitude range. The detector simulated here is a Faraday cup. The general geometry is similar to that of Havnes et al. (1996), Gelinas et al. (1998), Lynch et al. (2005) or Rapp et al. (2005). The simulated detector is radially symmetric with a radius of $R_{D}=40 \mathrm{~mm}$ and a depth of $80 \mathrm{~mm}$ (Fig. 1a). The detecting surface is at the bottom. To shield the detecting surface from ambient electrons and light ions, an electric field is applied with two grids. The outermost grid is biased at $-6.2 \mathrm{~V}$ and hence forms a potential barrier for electrons and light negative ions, and collects light positive ions. The second grid is held at $6.2 \mathrm{~V}$. The aerodynamic effect of the two shielding grids is negligible (Gumbel, 2001b). The detection surface is held at payload potential $(\sim 0 \mathrm{~V})$. The modelled smoke particles have a large mass compared to the electrons and light ions such that their kinetic energy is sufficient to make them largely unaffected by the electric potential. The charged particles are assumed to be either positively or negatively charged by one elementary charge $( \pm e)$. The electric field was modelled by SIMION 3-D version 6.0 computer software (Dahl, 1995) and the acceleration/deceleration of the charged particles in the electric

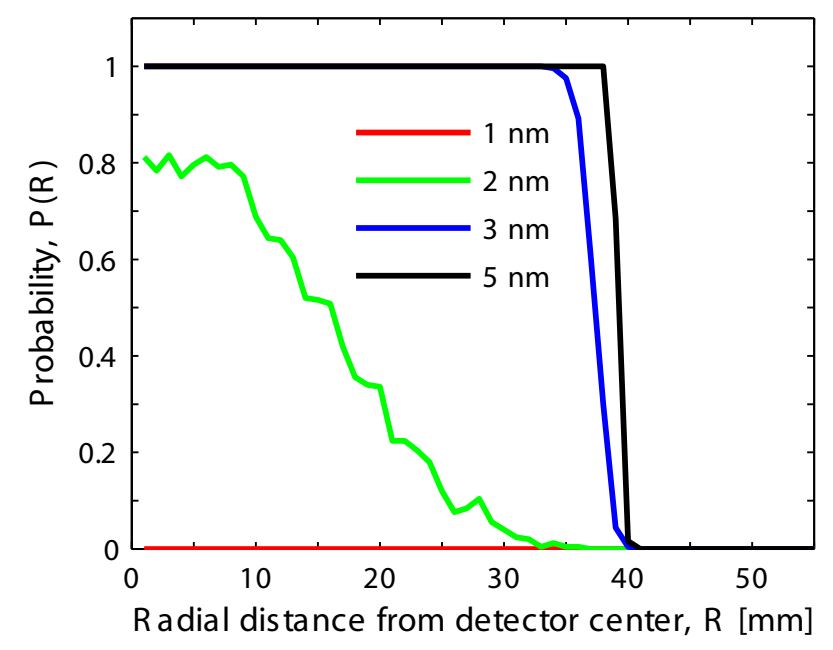

Fig. 4. Detection probability for $1,2,3$ and $5 \mathrm{~nm}$ radius positively charged particles with the Brownian motion model for the unventilated detector at an altitude of $85 \mathrm{~km}$.

field is then determined from multiplying the electric field strength with $\pm e$ and dividing with the particle mass $m_{p}$. Three designs of the detector were modelled, one without any ventilation holes (Fig. 1a) and two with holes (Figs. 1b and c) in the detection surface to let air flow through the detector. The holes give a transmission of $10 \%$ and $50 \%$ through the detection surface, respectively. Their purpose is to improve the aerodynamic properties of the detector, thus making it possible to detect smaller particles than with the unventilated detector.

\subsection{Impact simulations}

In order to compare different model runs, an effective relative cross section $\sigma_{\text {eff }}$ is defined as the ratio between the real impact cross section and the geometrical cross section of the detector $\sigma_{\text {geom }}=\pi R_{D}^{2}$

$\sigma_{\text {eff }}=\frac{\sigma_{\text {real }}}{\sigma_{\text {geom }}}$.

The real impact cross section is defined as

$\sigma_{\text {real }}=\int_{0}^{\infty} P(R) d A$,

where $P(R)$ is the probability with which a particle incident at a distance $R$ from the symmetry axis will impact on the detector surface area $A$. Figure 4 shows the impact probability for various particle sizes at an altitude of $85 \mathrm{~km}$ as simulated by the Brownian model. Particles are introduced in the gas flow in the undisturbed region in front of the detector and at different radial distances $R$ from the symmetry axis (Figs. 2 and 3). The particles are then traced towards the detector and registered if they impact on the detection surface. For 


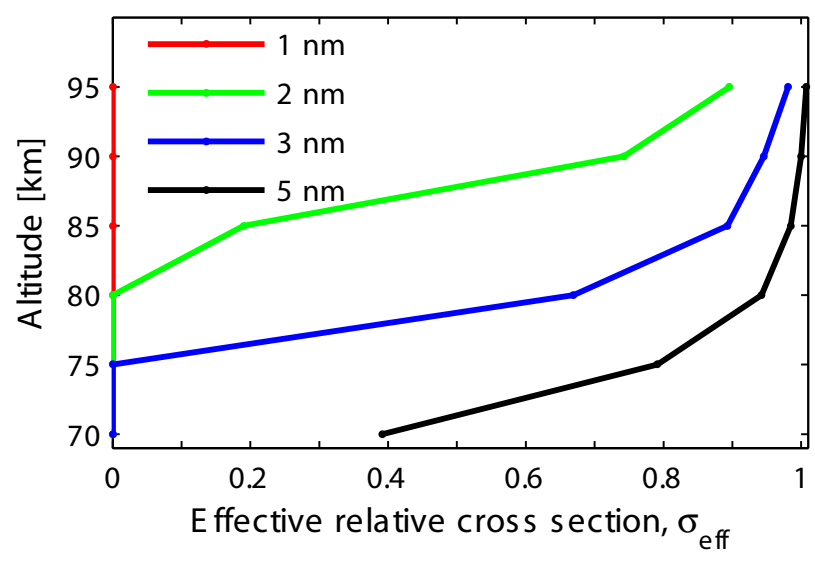

Fig. 5. Effective relative cross section for positively charged particles of different radii at different altitudes. Unventilated detector design.

the statistical Brownian motion model several particles are traced from each start position and for each particle radius $r_{p}$, and a detection probability $P(R)$ is determined. For the case in Fig. 4 typically 250 particles were traced with the Brownian motion model for each start position and particle radii 1, 2, 3 and $5 \mathrm{~nm}$. Figure 4 shows that none of the $1 \mathrm{~nm}$ particles hit the detection surface at this altitude for any start position, whereas $2 \mathrm{~nm}$ particles are detected with up to $80 \%$ probability. Larger particles are largely detected if their start positions lie within the geometric cross section of the detector. Figure 5 shows the effective relative cross section for various particle sizes as a function of altitude. These simulations were performed with the Brownian model for positively charged particles and the unventilated detector. Particles of $2 \mathrm{~nm}$ radius impact on the detection surface for altitudes around $85 \mathrm{~km}$ and above, whereas $1 \mathrm{~nm}$ radius particles do not impact at all.

\subsection{Brownian vs. continuous motion}

In the Brownian motion model the particles are embedded in the air flow and take part in the random molecular motion on their way towards the detector. As a comparison between Figs. 2 and 3 shows, this Brownian motion can have a decisive influence on the detection of smoke particles. This is also illustrated in Fig. 6 that compares the Brownian motion results (blue lines) from Fig. 5 with the continuous motion results (red lines). From this comparison we can see that the Continuum motion model overestimates the number of detected particles for the two smallest simulated particle sizes at the higher altitudes. In other words, the continuum motion model predicts a too low altitude for the transition from low to high detection probability for a given particle size. As expected, this discrepancy between the two models decreases with increasing particle size when Brownian motion becomes less and less important.
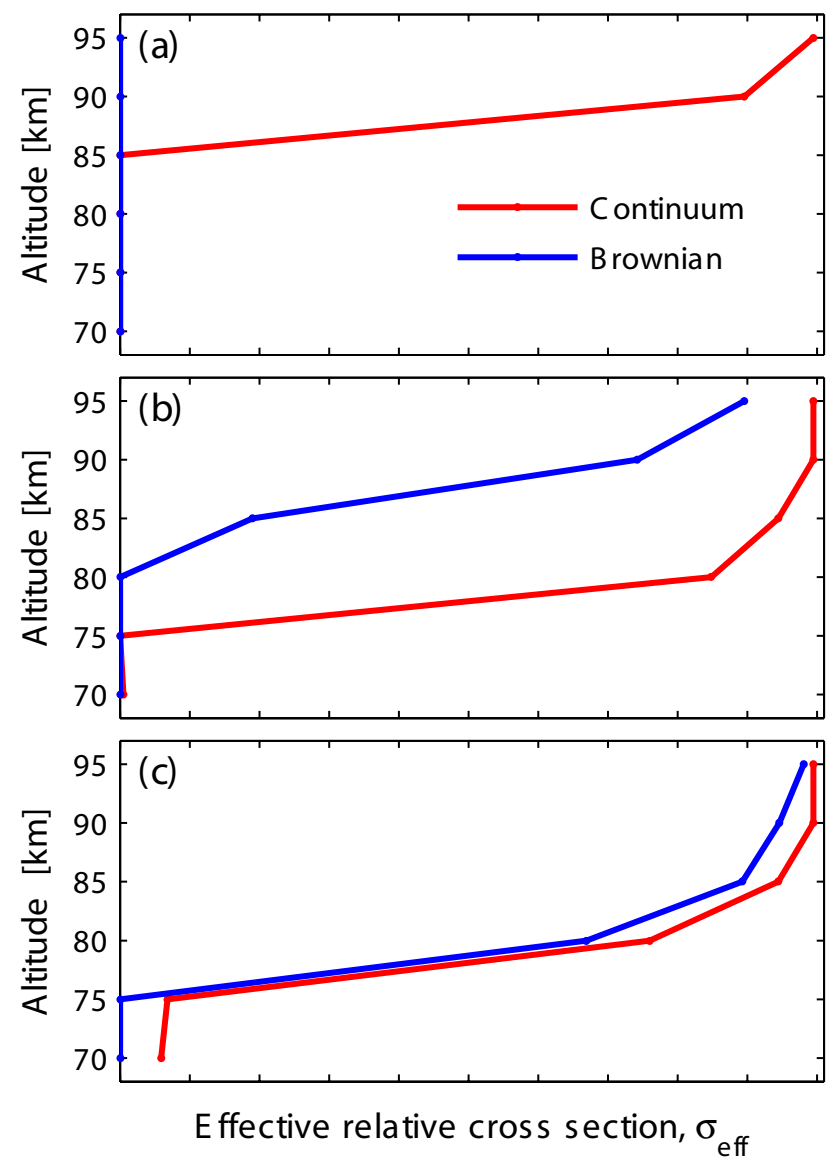

Fig. 6. Comparison between the effective relative cross section determined with the Continuum motion model and the Brownian motion model for (a) $1 \mathrm{~nm}$, (b) $2 \mathrm{~nm}$, and (c) $3 \mathrm{~nm}$ radius positively charged particles and the unventilated detector design.

The assumption of a continuum drag coefficient $C_{D}$ (Eq. 3) in the Continuum motion model is valid for larger particles (larger than $3 \mathrm{~nm}$ ), but should not be used for smaller meteoric smoke particles. The smallest particles can be completely decelerated by the stagnating air flow inside the detector. Their further motion is then governed by a Brownian diffusion. This random behaviour cannot readily be described by a continuous motion model. All following simulations are based on the Brownian motion model.

\subsection{Detection efficiencies}

In Fig. 7 we compare the three detector designs of Fig. 1, one without any holes for ventilation (red line) and the other two with venting holes in the detection surface for $10 \%$ (blue line) and 50\% (black line) transmission, respectively. Only the particles that actually hit the ventilated detector surface are included, not the total number of particles that reach the back of the detector including those that go through the venting holes. Except for the ECOMA detector by Rapp et 

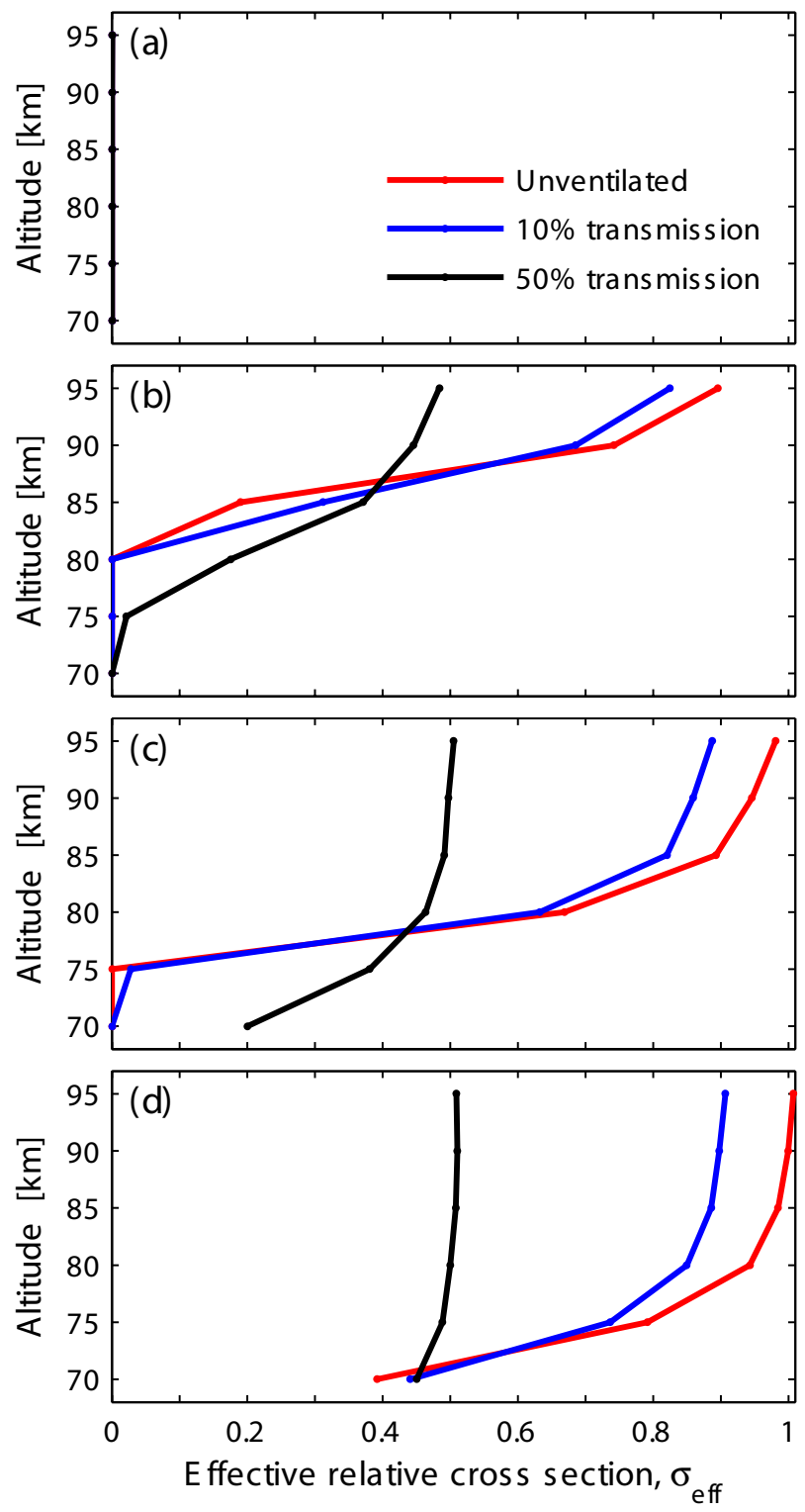

Fig. 7. Comparison between the effective relative cross section for the unventilated detector design and the two ventilated detector designs with $10 \%$ and $50 \%$ transmission, respectively, for (a) $1 \mathrm{~nm}$, (b) $2 \mathrm{~nm}$, (c) $3 \mathrm{~nm}$, and (d) $5 \mathrm{~nm}$ radius positively charged particles.

al. (2005), Faraday detectors have so far been designed without any holes for ventilation. From the figure we see that at high altitudes, for all particle radii, there is a detection difference of approximately $10 \%$ (50\%) between the unventilated and the ventilated detector with the $10 \%$ (50\%) transmission. For the rarefied conditions at these altitudes, these ventilation holes do not have a significant effect on the flow field inside the detector and, hence, these differences in detection probability simply reflect the geometric transmission differences through the detector surfaces. At lower altitudes, on the other hand, the holes result in an enhanced airflow and in
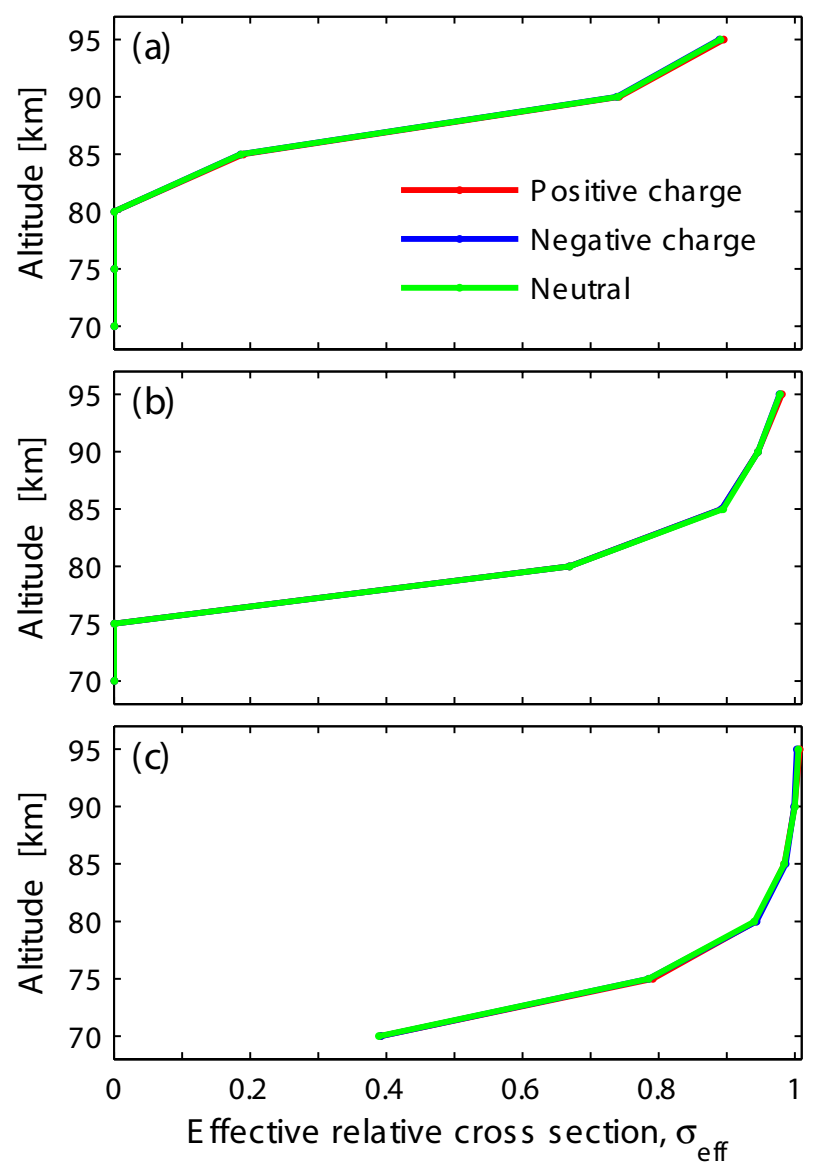

Fig. 8. The different effective relative cross sections for positive, negative and neutral particles for (a) $2 \mathrm{~nm}$, (b) $3 \mathrm{~nm}$, and (c) $5 \mathrm{~nm}$ radius particles and the unventilated detector design.

a decreased air number density inside the detector compared to the unventilated one (Fig. 1). As a result, one could expect that the effective relative cross-section increases and that a detection of smaller particles becomes possible at lower altitudes. However, for the ventilated detector design with $10 \%$ transmission, we do not observe this effect with the Brownian motion model. On the other hand, with the ventilated design with 50\% transmission (Fig. 1c), this effect can be seen. The detection of the 2 and $3 \mathrm{~nm}$ radius particles (Fig. $7 \mathrm{~b}$ and c) can be extended to lower altitudes by this detector design as compared to the other two. To summarise, in order to extend the detection of smaller particles to lower altitudes, a ventilated design with $10 \%$ transmission is not sufficient but a transmission of $50 \%$ provides a significant improvement.

While electrons and light ions will be stopped, the applied electric field in the detector should not affect particles of $1 \mathrm{~nm}$ radius and larger, since their kinetic energy is large enough to get them through the potential barrier. To determine whether the detection ability is charge-dependent, trajectories for both positively and negatively charged particles were modelled towards the detector. Figure 8 shows 
that there is no bias towards detecting a larger fraction of neutral (green line), positive (red line) or negative (blue line) particles. The combination of the two oppositely charged shielding grids thus affects positively and negatively charged particles in the same way for the particle sizes considered here.

\section{Conclusion and outlook}

Rocket-borne in-situ detection is the most direct way to obtain information about the mesospheric smoke particle layer. We have here performed detailed simulations of the detection process for a typical probe for charged particles. The detection efficiency for meteoric smoke particles in the nanometre size range is very much altitude dependent. Below $\sim 70$ $75 \mathrm{~km}$ it is difficult to detect particles at all for the sizes considered here. Particles are expected to be larger at lower altitudes (Megner et al., 2006), but nonetheless our simulations suggest an aerodynamic lower limit for the rocket-borne impact detection of smoke.

As illustrated in Fig. 6, Brownian motion is very important, especially for the smallest dust particles. The smallest particles can be completely decelerated by the stagnating airflow inside the detector and their further motion is then governed by a Brownian diffusion. This random behaviour is difficult to describe in Continuous motion models. The momentum transfer cannot be regarded as continuous and approaches based on a continuum drag coefficient (Eq. 3) should be avoided. For the smoke particle sizes simulated here, the use a Brownian motion model is mandatory to correctly describe the statistical motion near the Faraday cup detector.

Figure 7 sumarizes the difference between the three detector designs considered here. The open design with a transmission of $10 \%$ is not sufficient for an enhanced air flow through the detector and the air flow stagnates. With a transmission of 50\%, however, the airflow through the detector is enhanced and it becomes possible to extend the detection of the simulated 2 and $3 \mathrm{~nm}$ radius particles to lower altitudes.

While keeping the ionospheric plasma out, the shielding grids do not have a major influence on the detection of charged nanometre size particles. Figure 8 shows that there is no tendency for particles of different charge to be detected with different efficiencies. There is no general difference between neutral and charged particles for particle sizes down to $1 \mathrm{~nm}$ and only small ions and electrons from the ambient plasma will be stopped from entering the inner part of the detector.

For the future, we consider a number of improvements to our particle flow model. The Brownian motion description can be further developed to include a more realistic treatment of the molecule/particle collision. Both inelastic collisions and non-spherical smoke particles can be considered. The density of smoke particles is not known but normally assumed to be similar to the density of meteorites (ordinary chondrites $2-3 \mathrm{~g} / \mathrm{cm}^{3}$ ). If the density is smaller, e.g. porous particles, the aerodynamic effects become larger. As mentioned earlier, the mass loss due to heating and subsequent sublimation in the shocked gas flow is negligible for smoke particles. The mass loss for large ice particles (NLC) is also small, but becomes very important for smaller ice particles $(<10 \mathrm{~nm})$ (Horányi et al., 1999) and must be included in the model if ice particles are to be traced. Also neglected are effects of payload charging by photons, ions or particles as well as the possibility that incident particles trajectories are influenced by such a payload charging (Sternovsky et al., 2004). Finally, the simulations can be extended into three dimensions, e.g. in order to simulate rocket payloads with angles of attack other than $0^{\circ}$ or payloads that are asymmetric.

Regarding meteoric smoke particles, important questions concern their number density and size distribution as a function of altitude as well as the fraction of charged particles. Therefore, we need quantitative ways to relate measured particle populations to the real particle populations in the atmosphere. In this paper we have shown how the sizedependent, altitude-dependent and charge-dependent detection efficiency can be determined for a given instrument design. We have thus taken an important step towards a better understanding of the detection of meteoric smoke particles. However, there remain many open questions such as particle/surface interactions, secondary charge generation, and payload charging. After 50 years of ionospheric rocket studies, surprisingly many open questions remain on these issues. Further model developments are highly desirable that in a consistent way combines aerodynamics, the flow of particles and charges as well as electric fields and payload potentials.

\section{Appendix A}

As mentioned in Sect. 2.3 the molecule/particle collisions can be regarded as binary in rarefied gas conditions just involving one particle and one molecule. The collisions in the Brownian motion model are treated as elastic, i.e. there is no interchange of internal energy.

\section{A1 Post-collision velocity}

The momentum and energy equations for the molecule/particle collision can be written as

$$
\begin{aligned}
& m_{g} \boldsymbol{v}_{g}+m_{p} \boldsymbol{v}_{p}=m_{g} \boldsymbol{v}_{g}^{\prime}+m_{p} \boldsymbol{v}_{p}^{\prime}=\left(m_{g}+m_{p}\right) \boldsymbol{v}_{m} \\
& m_{g} v_{g}^{2}+m_{p} v_{p}^{2}=m_{g} v_{g}^{\prime 2}+m_{p} v_{p}^{\prime 2} .
\end{aligned}
$$

Here $m_{g}, \boldsymbol{v}_{g}$ and $m_{p}, \boldsymbol{v}_{p}$ are the masses and pre-collision velocities of the molecule and particle, respectively. The primed velocities are the post-collision velocities, and $\boldsymbol{v}_{m}$ is the velocity of the centre of mass of the two collision partners

$\boldsymbol{v}_{m}=\frac{m_{g} \boldsymbol{v}_{g}+m_{p} \boldsymbol{v}_{p}}{m_{g}+m_{p}}$ 
The relative velocities between the air molecule and the dust particle before and after the collision are

$\boldsymbol{v}_{\text {rel }}=\boldsymbol{v}_{g}-\boldsymbol{v}_{p}$

$\boldsymbol{v}_{\text {rel }}^{\prime}=\boldsymbol{v}_{g}^{\prime}-\boldsymbol{v}_{p}^{\prime}$

Combining Eqs. (A1), (A3) and (A4), the pre-collision velocities can be described as

$\boldsymbol{v}_{g}=\boldsymbol{v}_{m}+\frac{m_{p}}{m_{g}+m_{p}} \boldsymbol{v}_{\text {rel }}$,

$\boldsymbol{v}_{p}=\boldsymbol{v}_{m}-\frac{m_{g}}{m_{g}+m_{p}} \boldsymbol{v}_{\mathrm{rel}}$

and similarly for the post-collision velocities. Equation (4) gives the post-collision velocity of the particle. The precollision velocities relative the centre of mass are then $\boldsymbol{v}_{g}-\boldsymbol{v}_{m}$ and $\boldsymbol{v}_{g}-\boldsymbol{v}_{m}$. Combining Eqs. (4), (A2) and (A5) it can be shown that the magnitude of the relative velocity is unchanged by the collision, i.e. $v_{\text {rel }}^{\prime}=v_{\text {rel }}$. Both $v_{m}$ and $v_{\text {rel }}$ can be calculated from the pre-collision velocities and, thus, the determination of the post-collision velocities reduces to the calculation of the change in direction of the relative velocity vector.

After a molecule has collided with a particle, the particle will have a new velocity $\boldsymbol{v}_{p}^{\prime}$, according to Eq. (4), with

$\boldsymbol{v}_{\text {rel }}^{\prime}=v_{\text {rel } \boldsymbol{e}} \hat{\boldsymbol{e}}=\left|\boldsymbol{v}_{g}-\boldsymbol{v}_{p}\right| \hat{\boldsymbol{e}}$.

For elastic collisions between spherical collision partners, the post-collision relative velocity is distributed isotropically with a random direction

$\hat{\boldsymbol{e}}=\left[\begin{array}{c}\sin \theta \cos \phi \\ \sin \theta \sin \phi \\ \cos \theta\end{array}\right]$

with the angles $\theta$ and $\phi$ determined from

$\theta=\arccos \left(2 r_{1}-1\right)$

$\phi=2 \pi r_{2}$

where $r_{1}$ and $r_{2}$ are random numbers between 0 and 1 .

A2 Statistical collision time and mean relative speed

To determine the probability for collision at a certain time we need the mean collision time between two collisions

$\bar{\tau}_{\mathrm{coll}}=\frac{1}{\sigma N_{g} \bar{v}_{\mathrm{coll}}}$

Here $N_{g}$ is the air number density at a specific point, $\bar{v}_{\text {coll }}$ is the mean collision speed, and $\sigma$ is the collision cross section

$\sigma=\pi\left(r_{g}+r_{p}\right)^{2}$

where $r_{g}=0.185 \mathrm{~nm}$ is the effective radius of an air molecule and $r_{p}$ is the radius of the particle. The statistical collision time is then given by Eq. (6). The mean collision speed $\bar{v}_{\text {coll }}$ between a particle and the air molecules in the gas flow is difficult to determine. It can be calculated by integrating over the components of the molecular velocity distribution

$$
\begin{gathered}
\bar{v}_{\text {coll }}=\iint_{-\infty}^{\infty} \int\left|\left(\overline{\boldsymbol{V}}_{g}+\boldsymbol{v}_{\mathrm{gth}}\right)-\boldsymbol{v}_{p}\right| f\left(u_{\mathrm{gth}}\right) \\
f\left(v_{\mathrm{gth}}\right) f\left(w_{\mathrm{gth}}\right) d u_{\mathrm{gth}} d v_{\mathrm{gth}} d w_{\mathrm{gth}}
\end{gathered}
$$

with the Maxwellian distribution function for the thermal velocity component $u_{\text {gth }}$

$f_{0}\left(u_{\mathrm{gth}}\right)=\left(\frac{m_{g}}{2 \pi k_{B} T_{g}}\right)^{\frac{1}{2}} \exp \left[-\frac{m_{g} u_{\mathrm{gth}}^{2}}{2 k_{B} T_{g}}\right]$

and correspondingly for the $v_{\text {gth }}$ and $w_{\text {gth }}$ components. Equation (A11) can be simplified to

$\bar{v}_{\text {coll }}=\frac{\bar{v}_{\text {gth }}}{2 a} \times \int_{0}^{\infty} x^{2} \exp \left(-(x-a)^{2}\right)-\exp \left(-(x+a)^{2}\right) d x$

where $\bar{v}_{\text {gth }}$ is the mean thermal velocity and $a$ describes the relative speed between particle and mean gas flow:

$a=\frac{2}{\sqrt{\pi}} \times \frac{\left|\boldsymbol{v}_{p}-\overline{\boldsymbol{V}}_{g}\right|}{\bar{v}_{\mathrm{gth}}}$.

The mean collision speed is then calculated for each new particle position, and a random collision time is chosen by Eq. (6) and collisions are performed accordingly.

Acknowledgements. We thank I. Strelnikova for providing the pre-calculated electric field for the Faraday cup type detector from the SIMION 3D version 6.0 software. J. Hedin thanks for the support from the Swedish National Graduate School of Space Technology.

Edited by: P. Hartogh

\section{References}

Bird, G. A.: Aerodynamic effects on atmospheric composition measurements from rocket vehicles in the thermosphere. Planet. Space Sci., 36(9), 921-926, 1988.

Bird, G. A.: Molecular Gas Dynamics and the Direct Simulation of Gas Flows, Oxford University Press, Oxford, 1994.

Ceplecha, Z., Borovicka, J., Elford, W. G., ReVelle, D. O., Hawkes, R. L., Porubcan, V, and Simek, M: Meteor phenomena and bodies, Space Sci. Rev., 84, 327-471, 1998.

Croskey, C., Mitchell, J., Friedrich, M., Torkar, K., Hoppe, U.-P., and Goldberg, R.: Electrical structure of PMSE and NLC regions during the DROPPS program, Geophys. Res. Lett., 28, 14271430, 2001.

Dahl, D. A.: SIMION 3D: version 6.0, Ion Source Software, KLACK Inc., Idaho Falls, 1995. 
Gabrielli, P., Barbante, C., Plane, J. M. C., Varga, A., Hong, S., Cozzi, G., Gaspari, V., Planchon, F. A. M., Cairns, W., Ferrari, C., Crutzen, P., Cescon, P., and Boutron, C. F.: Meteoric smoke fallout over the Holocence epoch revealed by iridium and platinum in Greenland ice, Nature, 432, 1011-1014, doi:10.1038/nature03137, 2004.

Gelinas, L. J., Lynch, K. A., Kelley, M. C., Collins, R. L., Baker, S., Zhou, Q., and Friedman, J. S.: First observation of meteoritic charged dust in the tropical mesosphere, Geophys. Res. Lett., 25, 4047-4050, 1998.

Gumbel, J.: Aerodynamic influences on atmospheric in situ measurements from sounding rockets, J. Geophys. Res., 106, 10553 $10563,2001 \mathrm{a}$.

Gumbel, J.: Rarefied gas flows through meshes and implications for atmospheric measurements, Ann. Geophys., 19, 563-569, 2001b.

Gumbel, J., Waldemarsson, T., Giovane F., Khaplanov, M., Hedin J., Karlsson B., Lossow, S., Megner L., Stegman J., Fricke, K. H., Blum, U., Voelger P., Kirkwood, S., Dalin. P. Sternovsky, Z., Robertson S., Horányi, M., Stroud, R., Siskind, D. E., Meier, R. R., Blum, J., Summers, M., Plane, J. M. C., Mitchell N. J., and Rapp, M.: The MAGIC rocket campaign - an overview, Proc. 17th ESA Symposium on European Rocket and Balloon Programmes and Related Research (ESA SP-590), 141-144, 2005.

Havnes, O., Troim, J., Blix, T., Mortensen, W., Naesheim, L. I., Thrane, E., and Tönnesen, T.: First detection of charged dust particles in the Earth's mesosphere, J. Geophys. Res., 101, 10839 $10847,1996$.

Hedin, A. E.: Extension of the MSIS Thermospheric Model into the Middle and Lower Atmosphere, J. Geophys. Res., 96, 11591172, 1991.

Hedin, J., Gumbel, J., and Rapp, M.: The aerodynamics of smoke particle sampling, Proc. 17th ESA Symposium on European Rocket and Balloon Programmes and Related Research (ESA SP-590), 2005.

Horányi, M., Gumbel, J., Witt, G., and Robertson, S.: Simulation of rocket-borne particle measurements in the mesosphere, Geophys. Res. Lett., 26, 1537-1540, 1999.

Hunten, D. M., Turco, R. P., and Toon, O. B.: Smoke and dust particles of meteoric origin in the mesosphere and thermosphere, J. Atmos. Sci., 37, 1342-1357, 1980.

Lanci, L. and Kent, D. V.: Meteoric smoke fallout revealed by superparamagnetism in Greenland ice, Geophys. Res. Lett., 33, L13308, doi:10.1029/2006GL026480, 2006.

Love, S. G. and Brownlee, D. E.: A direct measurement of the terrestrial mass accretion rate of cosmic dust, Science, 262, 550$553,1993$.

Lynch, K. A., Gelinas, L. J., Kelley, M. C., Collins, R. L., Widholm, M., Rau, D., MacDonald, E., Liu, Y., Ulwick, J., and Mace, P.: Multiple sounding rocket observations of charged dust in the polar winter mesosphere, J. Geophys. Res., 110, A03302, doi:10.1029/2004JA010502, 2005.

Mathews, J. D., Janches, D., Meisel, D. D., and Zhou, Q. H.: The micrometeroid mass flux into the upper atmosphere: Arecibo results and a comparison with prior estimates, Geophys. Res. Lett., 28, 1929-1932, 2001.

Megner, L., Rapp, M., and Gumbel, J.: Distribution of meteoric smoke - sensitivity to microphysical properties and atmospheric conditions, Atmos. Chem. Phys., 6, 4415-4426, 2006, http://www.atmos-chem-phys.net/6/4415/2006/.

Plane, J. M. C.: Atmospheric chemistry of meteoric metals, Chem. Rev., 103, 4963-4984, doi:10.1021/cr0205309, 2003.

Plane, J. M. C.: A time-resolved model of the mesospheric Na layer: constraints on the meteor input function, Atmos. Chem. Phys., 4, 627-638, 2004,

http://www.atmos-chem-phys.net/4/627/2004/.

Probstein, R. F.: Problems of Hydrodynamics and Continuum Mechanics, SIAM, 568-580, 1968.

Rapp, M. and Lübken, F.-J.: Modelling of particle charging in the polar summer mesosphere: part 1 - general results, J. Atmos. Sol. Terr. Phys., 63, 759-770, 2001.

Rapp, M., Gumbel, J., and Lübken F.-J.: Absolute density measurements in the middle atmosphere, Ann. Geophys., 19, 571-580, 2001, http://www.ann-geophys.net/19/571/2001/.

Rapp, M., Hedin, J., Strelnikova, I., Friedrich, M., Gumbel, J., and Lübken, F.-J.: Observations of positively charged nanoparticles in the nighttime polar mesosphere, Geophys. Res. Lett., 32, L23821, doi:10.1029/2005GL024676, 2005.

Rapp, M. and Thomas, G. E.: Modeling the microphysics of mesospheric ice particles - assessment of current capabilities and basic sensitivities., J. Atmos. Sol. Terr. Phys., 68, 715-744, 2006.

Rapp, M., Strelnikova, I., and Gumbel, J.: Meteoric smoke particles: evidence from rocket and radar techniques, Adv. Space Res., in press, doi:10.1016/j.asr.2006.11.021., 2007.

Robertson, S., Smiley, B., Sternovsky, Z., Gumbel, J., and Stegman, J.: Rocket-borne probes for charged ionospheric aerosol particles, IEEE Trans. Plasma Sci., 32, 716-723, 2004.

Rosinski, J. and Snow, R. H.: Secondary particulate matter from meteor vapors, J. Meteorol., 18, 736-745, 1961.

Schulte, P. and Arnold, F.: Detection of upper atmospheric negatively charged microclusters by a rocket borne mass spectrometer, Geophys. Res. Lett., 19, 2297-2300, 1992.

Smiley, B., Rapp, M., Blix, T. A., Robertson, S., Horányi, M., Latteck, R., and Fiedler, J.: Charge and size distribution of mesospheric aerosol particles measured inside NLC and PMSE during MIDAS MaCWAVE 2002, J. Atmos. Sol. Terr. Phys., 68, 114123, 2006.

Sternovsky, Z., Holzworth, R. H., Horányi, M., and Robertson, S.: Potential distribution around sounding rockets in mesospheric layers with charged aerosol particles, Geophys. Res. Lett., 31, L22101, doi:10.1029/2004GL020949, 2004.

Summers M. E. and Siskind, D. E.: Surface recombination of O and $\mathrm{H}_{2}$ on meteoric dust as a source of mesospheric water vapor, Geophys. Res. Lett., 26, 1837-1840, 1999.

Voigt, C., Schlager, H., Luo, B. P., Dörnbrack, A. Roiger, A., Stock, P., Curtius, J., Vössing, H., Borrmann, S., Davies, S., Konopka, P., Schiller, C., Shur, G., and Peter, T.: Nitric acid trihydrate (NAT) formation at low NAT supersaturation in polar stratospheric clouds (PSCs), Atmos. Chem. Phys, 5, 1371-1380, 2005.

von Zahn, U.: The total mass flux of meteoroids into the Earth's upper atmosphere, Proc. 17th ESA Symposium on European Rocket and Balloon Programmes and Related Research (ESA SP-590), 33-39, 2005. 